GEODESY AND CARTOGRAPHY

ISSN 2029-6991 print / ISSN 2029-7009 online

UDC 528.381

\title{
ATMOSPHERIC EFFECTS IN GEODETIC LEVELLING
}

\author{
Aleš Breznikar ${ }^{1}$, Česlovas Aksamitauskas \\ ${ }^{1}$ Faculty of Civil and Geodetic Engineering, University of Ljubljana, Jamova 2, SI-1000 Ljubljana, Slovenia \\ ${ }^{2}$ Department of Geodesy and Cadastre, Vilnius Gediminas Technical University, \\ Sauletekio al. 11, LT-10223 Vilnius, Lithuania \\ E-mails: ${ }^{1}$ ales.breznikar@fgg.uni-lj.si (corresponding author); ${ }^{2}$ ceslovas.aksamitauskas@vgtu.lt
}

Received 08 November 2012; accepted 12 December 2012

\begin{abstract}
The knowledge of physical processes and changes in the atmosphere is essential when addressing the fundamental problem, i.e. the accuracy of geodetic measurements. In levelling operations, all these changes are explained as the effect of refraction, which systematically distorts the results of levelling. Different ways of addressing the effect of refraction are represented based on the modelling of the vertical temperature gradient as the quantity that has the most influence on the refraction phenomenon.
\end{abstract}

Keywords: atmospheric refraction, geodetic levelling, model of vertical temperature gradient.

\section{Introduction}

Measurements in geodesy are mostly performed in the field, under real atmospheric conditions. These measurements are therefore subjected to all the changes that occur in the atmosphere. The atmosphere is a highly inhomogeneous medium, whose state is defined by a set of parameters that can quickly change, both spatially and temporarily.

Today, the errors resulting from environmental impacts are the main reason why the measurement accuracy is not even higher than it already is. The proper evaluation of the individual parameters occurring in the atmosphere, whose changes result in refraction effects, is the main issue. Studying the atmospheric effects in geodetic operations is an interdisciplinary activity, in which the problems are solved based on scientific achievements in relevant scientific areas.

In levelling operations, too, invar levelling rods and levelling instruments have reached a stage of development which would have permitted even higher accuracy, if it had not been limited by refraction. There are many problem domains where precise determination of the height difference between the stations on the Earth's surface is required. These problems are related to geophysics, geodynamics, engineering geodesy and, indeed, precise levelling.

Today, refraction represents the main source of systematic errors in levelling. Particularly, the effects of refraction are analysed as part of national levelling network campaigns. In most of developed countries, in the national first-order levelling surveys corrections for refraction effects are performed.

\section{Known characteristics of levelling refraction}

The principal knowledge about levelling refraction can be summarized as follows:

- On an inclined surface, the refraction systematically distorts the results of levelling;

- The amount of refraction error is proportional to the square of the length of the line of sight;

- The refraction effect increases with the increase of the temperature gradient;

- Under the influence of refraction, the height difference is reduced;

- If a certain levelling line is levelled under the same microclimatic conditions, the effects of refraction are not evident as deviations during the levelling in either direction, nor are they manifested as deviations in the closure of loops; rather it functions as the systematic error of the rod scale. The refraction error is manifested directly in the height error of individual benchmarks;

- The effect of humidity is minimal and can be, in most cases, neglected;

- On its way from the levelling instrument to the level rod, the horizontal line of sight is deflected upwards, which is the result of the negative temperature gradient in near-ground air layers.

The refractive index of air along the levelling line of sight changes as a function of the quantities causing it to change, hence resulting in the phenomenon of refraction (Mozzuchin 1999, 2010).

The refractive index in the atmosphere is mostly influenced by the following quantities: air temperature, air pressure, and content of humidity and carbon dioxide 
$\left(\mathrm{CO}_{2}\right)$. In ordinary levelling operations, mostly air mass in the range of $50 \mathrm{~cm}$ to $300 \mathrm{~cm}$ above ground is considered; hence the studying of the values and changes of the influencing quantities in these layers is meaningful.

The effect of air humidity is negligible since it represents, at the most, less than $1 / 20$ of the effect of temperature. The effect of carbon dioxide is even smaller.

Temperature and air pressure have the most significant effect on the refractive index in the air layers where levelling operations typically take place.

The amount of air pressure changes as a function of height. Generally speaking, air pressure decreases proportionally to the height above the ground. During the course of the day, the air pressure in a station changes depending on the general weather conditions. However, these changes of air pressure are slow and, in general, minimal. Evidently, the systematic effect of air pressure changes is eliminated in closed loops, while the effect of minor, random changes of air pressure is also negligible.

Temperature, along with its changes, is the main factor causing changes of the refractive index in the atmosphere. Hence the determination of the actual influence of levelling refraction depends on the use of as far as possible realistic models describing temperature gradients in the air layers where lines of sight are captured during levelling.

The changes of the refractive index are a function of both the absolute air temperature and the temperature differences of the air layers where lines of sight are captured. Here, we must distinguish between two terms, albeit the same quantity, i.e. temperature. The differences in temperature can be relatively large at low temperatures, and, vice versa, the differences can be minor at high temperatures, depending on different factors. Therefore they should be dealt with separately, i.e. absolute temperature and temperature gradients.

In almost all formulas for calculation of the correction for levelling refraction, the size of the refractive correction is proportional to the size of the temperature gradient. While the changes of other factors involved in levelling refraction do not significantly influence the change of refraction, the effect of temperature difference is great and can change the size of refractive effects by $100 \%$, and even $200 \%$. Temperature gradient in the elevations of up to $3 \mathrm{~m}$ above the ground, i.e. in the range of ordinary levelling operations, can be negative, positive, or equal to zero. Furthermore, temperature gradient cannot be considered as a random variable during the levelling operations.

The investigations to date have indicated that temperature gradient is the factor causing both random and systematic effects of refraction. The random part significantly affects the random error of levelling, while the systematic part will have grave effects on long lines. Hence, in precise measurements it is necessary to know the size of the temperature gradient.

In levelling lines with unsymmetrical slopes, i.e. the upward inclinations are smaller while the downward inclinations are larger, or vice versa, with the same height differences of rising and falling slopes, the effect of slope is reflected in the measured height of the levelling line.
This error also cannot be determined from the differences in levelling in either direction; however, its effect is evident in the closure of levelling loops. In the levelling loops that involve symmetrical slopes, the refraction error will not be expressed as an error in levelling loop closure. However, the effects of the part of the refraction error resulting from the meteorological conditions during levelling will not be negligible. In levelling loops involving unsymmetrical slopes, the refraction error can be eliminated only partly. The non-eliminated part of the error results in non-closure of levelling loops, which is frequently higher that the allowed deviation.

\section{Ways of addressing the problem of refraction}

There are several different approaches to the determination of levelling refraction. Of the existing models solving the problem of levelling refraction, one can distinguish between three main groups (Heer 1983; Brunner 1984):

- Direct refraction models in which the corrections for refraction effect are calculated by taking into account the measured temperature differences and the selected equations for temperature changes near ground level, or, alternatively, the temperature changes serve for temperature gradient quantification. The temperature gradient is the input of the model describing the physical changes in the atmospheric boundary layers.

- Indirect refraction models where the corrections for refraction are also calculated based on the size of the temperature gradient. In these cases, the temperature gradient is expressed in relation to meteorological and geometric parameters, as a function of height above the ground, tilted surface, zenith distance of the Sun and the height variation of heat fluxes. In this case also, the temperature gradient can be calculated based on the measurements of temperature.

- The statistical analysis of the levelling operations can estimate the individual types of errors; hence it is possible to assume the size of the error due to the effects of refraction.

\subsection{Models of levelling refraction based on measurements of temperature differences}

The models for refraction error calculation originate from the common basis, which is expressed as the effect of the temperature gradient on the refractive index of air and the assumption that the isothermal layers are parallel to the ground (Kakkuri 1984). The individual models differentiate in the different models for temperature change $\Delta t$ and are named after their authors:

$$
\begin{aligned}
& \text { Kukkamaeki: } \Delta t=b\left(z_{2}^{c}-z_{1}^{c}\right), \\
& \text { Reissmann: } \Delta t=b\left(z_{2}-z_{1}\right)+c\left(z_{2}^{2}-z_{1}^{2}\right), \\
& \text { Lallemand } \Delta t=b\left(\log \left(z_{2}+c\right)-\log \left(z_{1}+c\right)\right),
\end{aligned}
$$

where $\Delta t$ - change of temperature; $z_{2}, z_{1}$ - height above ground; $b, c$-coefficients obtained by measurements of temperature at different heights. 
The above mentioned models for temperature changes are incorporated into the equation describing the refraction effect as a function of temperature changes:

$$
r_{s, z}=-\operatorname{ctg}^{2} \gamma D \int_{z_{i}}^{z_{s, z}} \Delta t d z
$$

where $z_{i}$ - height at the instrument; $z_{s, z}$ - height at the rod, i.e. front and back; $D$ - factor of the effects of temperature changes on the refractive index; $g$ - slope of terrain; $r_{s, z}$ - effect of refraction.

Another possibility of addressing the problem of refraction with measured temperature differences is to model the temperature gradient. For the first time, this model was approximated by Brock in the following form (Heer 1983):

$$
\frac{d t}{d z}=a z^{b},
$$

temperature gradient at a height $z=1 \mathrm{~m}, z$ - height above ground.

The value of parameter $a$ changes during the course of the day and, indeed, during the course of the year. In his studies, Brocks found that within the height range associated with levelling lines of sight, the value of $b=-1$ can be assumed.

Parameter $a$ can be also obtained by measurements of temperature differences:

$$
a=\frac{t_{2}-t_{1}}{\ln \frac{z_{2}}{z_{1}}}
$$

where $t_{2}, t_{1}$ - temperatures measured at heights $z_{2}$ and $z_{1}$.

Based on these findings, the equation of the refraction effect can be written as follows:

$$
r_{s, z}=\frac{c_{1} s^{2}}{2}+\frac{c_{2} s^{2}}{z_{1}-z_{s, z}} a\left[1-\frac{z_{s, z}}{z_{i}-z_{s, z}} \ln \frac{z_{i}}{z_{s, z}}\right]
$$

where $s$ - length of the line of sight.

If we have records of multiple temperature differences at the heights transected by the levelling line of sight, these temperature differences can be directly used in the equation calculating the refraction effect. In such cases, it is not necessary to know the coefficients in the temperature equations, but rather the problem is solved using approximate integration, i.e. summing. The accuracy of the method depends on the number of stations with known temperature differences. The refraction effect on the measured height difference can be calculated using the summing method as follows:

$$
r_{s, z}=-\operatorname{ctg}^{2} \gamma D \sum_{z i} \Delta t \Delta z
$$

where $\Delta z$ - height difference between two temperature differences; $\Delta t$ - temperature difference between the instrument and the individual point of line of sight.

\subsection{Indirect model of correction for effects of refraction}

In previous equations addressing levelling refraction, the equations were expressed in units of gradient refractivity as a function of the temperature gradient. The temperature gradient can be expressed in units of meteorological parameters as a function of height above ground, i.e. the assumed temperature function.

$\mathrm{K}$. Webb was the first to show that it would, in fact, be beneficial to calculate the temperature gradient within the boundaries of meteorological parameters. The equations were derived in the framework of comprehensive studies of atmospheric turbulence and heat balance in the boundary layers. These equations, which arise from a simple basic theory, were developed using highly complex and precise meteorological measurements.

$$
\begin{aligned}
& r_{s, z}=-10^{-6} \frac{P}{T^{2}} s^{2} \times \\
& \left(1-3.3 H^{2 / 3}\left(\frac{z_{i}^{2 / 3}+2 z_{i}^{-1 / 3} z_{s, z}-3 z_{s, z}^{2 / 3}}{\left(z_{i}-z_{s, z}\right)^{2}}\right)\right),
\end{aligned}
$$

where $P$ - preasure in [mbar]; $T$ - temperature of the air in $[\mathrm{K}]$.

The key quantity in the equation above is heat flux $(H)$, which represents the quantity of heat energy carried by convective heat flow.

Heat flux is one of the most characteristic meteorological parameters; however, it cannot be measured directly. Angus-Leppan (1984) proposed three ways of determining heat flux:

- Determination of the components of heat flux by means of the heat balance equation;

- Determination of the heat flux based on the relation between the basic atmospheric parameters, such as cloudiness, effects of wind and humidity, and the Sun's zenith distance;

- Heat flux can be determined from measurements of temperature differences using the equation describing the temperature distribution in the lower layers of the atmosphere. If the equation is integrated between two different levels, and the potential temperature is replaced by the actual temperature, we obtain:

$$
0.078 H^{2 / 3}=\frac{\left(t_{2}-t_{1}\right)+0.01\left(z_{2}-z_{1}\right)}{z_{2}^{-1 / 3}-z_{1}^{-1 / 3}} .
$$

\subsection{Statistical analysis}

The statistical analysis of levelling results suggests the presence of both random and systematic errors. The corrections for refraction effects in levelling operations try to eliminate the systematic part of the errors due to refraction; however, it is recommended that the results of levelling are statistically processed, while trying to determine the ratio of the systematic error to the total error. 
The determination of levelling refraction from adjustments was suggested by Remmer (1982a, 1982b, 1983). For the analysis, he used the results of the second levelling campaign of Finland (1935-1937), which was very precise. Instead of assuming the refraction correction based on measurements of temperature, he proposed the use of equations of the observations containing correction for refraction.

However, the statistical processing of the levelling results, as proposed by Remmer, has two deficiencies regarding the refraction error:

- It addresses the refraction error which is manifested only in specific conditions of levelling operations: the closure of levelling loops and deviations during the levelling in either direction. However, in this way the refraction error which is manifested directly in the height error of individual stations is not cancelled out. Hence, the entire systematic effect of the refraction error cannot be eliminated.

- Furthermore, all errors obtained in the statistical analysis cannot be associated with the effects of refraction. In the way of data processing, other systematic effects occur during levelling operations.

\section{Conclusions}

The effect of vertical refraction in levelling operations is not negligible and represents a major part of systematic errors in levelling. Temperature gradient is the main factor influencing the size of refraction, while the effect of other factors is negligible, since they represent a minimal proportion of refraction effects. Hence the correct estimation of the size and changes of the temperature gradient is the most important task in quantifying the effect of refraction.

The corrections for refraction effect calculated using the chosen model of temperature gradient are a good solution to the problem of refraction; however, additional measurements of major temperature differences during levelling are required, prolonging levelling operations.

In indirect calculations of refraction effects, extensive studies to elaborate sufficiently accurate models are needed, providing satisfactory descriptions of the different atmospheric conditions. The advantage is that the complex measurements of temperature differences are not necessary. However, the method requires detailed meteorological data on cloudiness, wind, Sun elevation and vegetation. The collection of these data can also be time-consuming.

\section{References}

Angus-Leppan, P. V. 1984. Refraction in geodetic levelling, in F. K. Brunner (Ed.). Geodetic Refraction. Berlin: SpringerVerlag, 163-180.

Brunner, F. K. 1984. Modelling of atmospheric effects on terrestrial geodetic measurement, in F. K. Brunner (Ed.). Geodetic Refraction. Berlin: Springer-Verlag, 143-162.

Heer, R. 1983. Applications of different refraction models on measuring results of the Levelling Test Loop Koblenz, in H. Pelzer (Ed.). Workshop Precise Levelling. Duemmlers Verlag, 251-281.

Kakkuri, J. 1984. About the future use of the Kukkamaki levelling refraction formula, in H. Pelzer (Ed.). Workshop Precise Levelling. Duemmlers Verlag, 235-241.

Mozzuchin, O. A. 1999. Einige Aspekte zum Problem der geodaetischen Refraktion, Vermessungswesen und Raumordnung 61(3): 172-175.

Mozzuchin, O. A. 2010. Zur Analyse von Nivellementergebnissen, Allgemeine Vermessungs Nachrichten 3: 110-112.

Remmer, O. 1982a. Non-random effects in the Finnish levellings of high precision, Manuscripta Geodaetica 353-373.

Remmer, O. 1982b. Modellings errors in geometric levelling, Vermwes 7: 355-372.

Remmer, O. 1983. Refraction and other systematic effects in levelling, in H. Pelzer (Ed.). Workshop: Precise Levelling. Duemmlers Verlag, 181-191.

Aleš BREZNIKAR. Assoc. Prof., Dr at the Faculty of Civil and Geodetic Engineering, University of Ljubljana, Jamova 2, SI-1000 Ljubljana, Slovenia. $\mathrm{Ph}+38641$ 356718, e-mail: ales.breznikar@fgg.uni-li.si.

The autor of more than 20 scientific papers participated in a number of international conferences.

Research interests: engineering surveying, measuring systems

Česlovas AKSAMITAUSKAS. Prof., Dr, Dept of Geodesy and Cadastre, Vilnius Gediminas Technical University, Sauletekio al. 11, LT-10223 Vilnius, Lithuania. Ph +370 52744703 , Fax +370 52744 705, e-mail: ceslovas.aksamitauskas@vgtu.lt.

Doctor (1982). Habilitation procedure in 2011. Author of two teaching books and more than 20 scientific papers. Participated in many intern conferences.

Research interests: geodetic instrumentation, automation of measurements, angular and distance measurements. 